\title{
Double reduction analysis of Benjamin, DGH and generalized DGH equations
}

\author{
Muhammad Danish Khan \\ Department of Mathematics, \\ School of Science and Engineering, \\ Lahore University of Management Sciences, Lahore, Pakistan \\ Email: 08070005@lums.edu.pk
}

\begin{abstract}
The exact solutions of non-linear evolution equation, Benjamin equation, Dullin-Gottwald-Holm (DGH) equation and generalized Dullin-Gottwald-Holm equation are established using the conserved vectors. The multiplier approach is applied to construct the conserved vectors for equations under consideration. For non-linear evolution equation three conserved vectors and for Benjamin equation four conserved vectors are obtained. The conserved vectors for DGH and generalized DGH equations were reported in [1]. The higher order multiplier is considered for DGH equation and a new conserved vector is found. The double reduction theory is utilized to obtain various exact solutions for Benjamin equation, DGH equation and generalized DGH equation.
\end{abstract}

\section{INTRODUCTION}

The association of symmetries (Noether, Lie-Bäcklund or non-local) with the conserved vectors plays an imperative role in the development of a new method for construction of exact solutions for partial differential equations (PDEs). The interested reader is referred to some important works in this direction. The classical Noether's theorem [2] provides a relationship between conservation laws and variational symmetries. Kara et al [3] derived the relationship between Lie-Bäcklund symmetries and conserved vectors. This idea of association was extended to non-local symmetries by Sjöberg [4], [5]. In [6], [7], Sjöberg developed the double reduction theory for PDEs with two independent variables and this theory based on symmetry conservation laws relationship. A $k^{t h}$ order PDE with two independent variables can be reduced to an ordinary differential equation (ODE) of order $k-1$ provided that at least one symmetry is associated with the conserved vector. Recently, Bokhari et al [9] generalized the double redaction theory for PDEs with $n$ independent variables.

This article deals with the exact solutions of $(1+1)$ dimensional non-linear evolution equation, Benjamin equation, DGH equation and generalized DGH equation. The non-linear evolution equation is a general form of some important equations like duffing equation, Landau-Ginburg-Higgs equation, sine-Gordon equation, $\phi^{4}$ equation, Klein-Gordon equation [9-16] etc. The multiplier approach also known as variational derivative approach is exploited to construct conservation laws for equations under consideration. Stuedel [17] introduced the multiplier approach and the conserved vectors were written in characteristic form as $D_{i} T^{i}=\Lambda^{\alpha} E_{\alpha}$. The determining equations for the multipliers (characteristics) were obtained by taking the variational derivative of $D_{i} T^{i}=Q^{\alpha} E_{\alpha}$ for the arbitrary functions not only for solutions of system of partial differential equations [18]. There are some other methods as well to obtain conservation laws. The comparison of different approaches to compute conservation laws for some PDEs was given in [19] (see also references therein). The symmetry conservation laws relationship is used to determine symmetries associated with the conserved vectors. Then the double reduction theory is applied to find exact solutions of under study equations. Some explicit solutions of DGH and generalized DGH equations are computed by utilizing the sine-cosine method $[27,28]$.

The arrangement scheme of this article is as follows. In Section 2, we define some basic definitions, the characteristic approach and double reduction theory. Conservation laws and exact solutions of non-linear evolution equation are discussed in section 3. Section 4 deals with the conservation laws and solutions of Benjamin equation. We construct conservation laws and exact solutions of DGH equation and generalized DGH equation in Sections 5 and 6 respectively. The concluding remarks are summarized at last.

\section{PRELIMINARIES}

The following definitions are well known [1], [3], [6], [7]. Suppose a $k^{\text {th }}$ order system of partial differential equations (PDEs)

$$
G_{\alpha}\left(x, u, u_{(1)}, u_{(2)}, \ldots, u_{(k)}\right)=0, \quad \alpha=1,2, \ldots, m,
$$

where $x=\left(x^{1}, x^{2}, \ldots, x^{n}\right)$ are the independent variables with coordinates $x^{i}$ and $u=\left(u^{1}, u^{2}, \ldots, u^{m}\right)$ the dependent variables with coordinates $u^{\alpha}$. In Eq. (1) $u_{i}$ symbolize the collection of $i$ th order partial derivatives i.e.

$$
u_{i}^{\alpha}=D_{i}\left(u^{\alpha}\right), \quad u_{i j}^{\alpha}=D_{j} D_{i} u^{\alpha}, \ldots,
$$

where

$$
D_{i}=\frac{\partial}{\partial x^{i}}+u_{i}^{\alpha} \frac{\partial}{\partial u^{\alpha}}+u_{i j}^{\alpha} \frac{\partial}{\partial u_{j}^{\alpha}}+\ldots,
$$

is known as the operator of total differentiation with respect to $x^{i}$.

The summation convention is adapted throughout for repeated indices.

The Lie-Bäcklund or generalized operator is defined as

$$
X=\xi^{i} \frac{\partial}{\partial x^{i}}+\eta^{\alpha} \frac{\partial}{\partial u^{\alpha}}+\sum_{s \geq 1} \zeta_{i_{1} \ldots i_{s}}^{\alpha} \frac{\partial}{\partial u_{i_{1} \ldots i_{s}}^{\alpha}},
$$


where $\xi^{i}, \eta^{\alpha} \in \mathcal{A}$ (space of differential functions). In Eq. (3) $\zeta_{i_{1} \ldots i_{s}}^{\alpha}$ are the additional coefficients and can be found from

$$
\begin{gathered}
\zeta_{i}^{\alpha}=D_{i}\left(W^{\alpha}\right)+\xi^{j} u_{i j}^{\alpha}, \\
\zeta_{i_{1} \ldots i_{s}}^{\alpha}=D_{i_{1}} \ldots D_{i_{s}}\left(W^{\alpha}\right)+\xi^{j} u_{j i_{1} \ldots i_{s}}^{\alpha}, \quad s>1 .
\end{gathered}
$$

A conserved vector of (1) is an $n$-tuple $T=$ $\left(T^{1}, T^{2}, \ldots, T^{n}\right), T^{i} \in \mathcal{A}, i=1,2, \ldots, n$, if

$$
D_{i} T^{i}=0 \text {, }
$$

holds for all solutions of (1).

The characteristic or multiplier $\Lambda^{\alpha}$ for conservation laws of system (1) satisfies the property [16], [17]

$$
\Lambda^{\alpha}\left(G_{\alpha}\right)=D_{i} T^{i}
$$

The determining equations for multipliers can be constructed by taking variational derivative on both sides of Eq. (6) (see [17])

$$
E_{u}\left[\Lambda^{\alpha}\left(G_{\alpha}\right)\right]=0,
$$

where $E_{u}$ is the Euler operator, for each $\alpha$, given by

$$
E_{u}=\frac{\partial}{\partial u^{\alpha}}+\sum_{s \geq 1}(-1)^{s} D_{i_{1}} \ldots D_{i_{s}} \frac{\partial}{\partial u_{i_{1} \ldots i_{s}}^{\alpha}}, \quad \alpha=1,2, \ldots, m .
$$

If $X$ and $T$ are the Lie-Bäcklund symmetry generator and conserved vector respectively of system (1) and if

$$
X\left(T^{i}\right)+D_{j}\left(\xi^{j}\right) T^{i}-T^{j} D_{j}\left(\xi^{i}\right)=0, \quad i=1,2, \ldots, n,
$$

then $X$ is said to be associated with $T$.

In case if $X$ is not associated with $T$, then the following theorem helps to find $T^{*}$, such that $X$ will be associated with $T^{*}$.

Theorem [20], [21]: Let $X$ is the Lie symmetry generator and $T$ is the conserved vector of (1), then

$$
T^{* i}=X\left(T^{i}\right)+T^{i} D_{j}\left(\xi^{j}\right)-T^{j} D_{j}\left(\xi^{i}\right), \quad i=1,2, \ldots, n,
$$

forms a new conserved vector of (1) which satisfies $D_{i} T_{i}^{*}=0$.

One can obtain a reduced conserved form of the $k^{\text {th }}$ order PDE (1) with two independent and one dependent variable by utilizing the associated symmetry along with the conserved vector of (1). In terms of similarity variables $r, s$ with the symmetry $X=\partial / \partial s$, the conservation laws can be expressed as

$$
D_{r} T^{r}+D_{s} T^{s}=0,
$$

where $T^{r}$ and $T^{s}$ in terms of old variables $(\mathrm{t}, \mathrm{x})$ are

$$
\begin{aligned}
T^{s} & =\frac{T^{t} D_{t}(s)+T^{x} D_{x}(s)}{D_{t}(r) D_{x}(s)-D_{x}(r) D_{t}(s)}, \\
T^{r} & =\frac{T^{t} D_{t}(r)+T^{x} D_{x}(r)}{D_{t}(r) D_{x}(s)-D_{x}(r) D_{t}(s)} .
\end{aligned}
$$

Since the conserved components $T^{s}$ and $T^{r}$ are functions of $\left(s, r, w, w_{r}, w_{r r}, \ldots, w_{r(k-1)}\right)$ for solutions under the action of $X$ and $T^{t}$ and $T^{x}$ are functions of $\left(t, x, u, u_{(1)}, u_{(2)}, \ldots, u_{(k-1)}\right)$, therefore Eq. (11) implies that

$$
\frac{\partial T^{s}}{\partial s}+D_{r} T^{r}=0
$$

which results in

$$
T^{r}=\int \frac{\partial T^{s}}{\partial s} d r+g(s)
$$

The association of $X$ with $T$ gives rise to $X T^{s}=0$ and $X T^{r}=0$, which further yields

$$
\frac{\partial}{\partial s} T^{r}=0 \quad \text { and } \quad \frac{\partial}{\partial s} T^{s}=0
$$

The conservation law in similarity variables reduces to

$$
D_{r} T^{r}=0 \text {. }
$$

Double reduction theorem [6]: A $k^{\text {th }}$ order PDE with two independent variables and one dependent variable which admits a symmetry generator $X$ and is associated with the conserved vector $T$ is reduced to a $(k-1)$ th order ODE i.e. $T^{r}=k$, where $T^{r}$ can be determined from the formula given in (13).

\section{CONSERVATION LAWS AND EXACT SOLUTION OF NON-LINEAR EVOLUTION EQUATION}

In this section, we will derive the conservation laws of a non-linear evolution equation by utilizing the multipliers approach, then using these conserved vectors we will work out for the exact solution of non-linear evolution equation using the double reduction theorem. The nonlinear evolution equation is

$$
u_{t t}+a u_{x x}+b u+c u^{3}=0,
$$

where $u=u(t, x)$ and $a, b, c$ are constants. Consider the multiplier of the form $\Lambda=\Lambda\left(t, x, u, u_{t}, u_{x}\right)$. The determining equations for multiplier of (15) are obtained by solving

$$
E_{u}\left[\Lambda\left(u_{t t}+a u_{x x}+b u+c u^{3}\right)\right]=0,
$$

where $E_{u}$ is the Euler operator described in (8).

Expansion of Eq. (16) gives rise to

$$
\begin{aligned}
& \Lambda_{u}\left(u_{t t}+a u_{x x}+b u+c u^{3}\right)-D_{t}\left[\Lambda_{u_{t}}\left(u_{t t}+a u_{x x}+b u+c u^{3}\right)\right. \\
& -D_{x}\left[\Lambda_{u_{x}}\left(u_{t t}+a u_{x x}+b u+c u^{3}\right)\right]+\Lambda\left(b+3 c u^{2}\right) \\
& +D_{t}^{2}[\Lambda]+D_{x}^{2}[a \Lambda]=0 .
\end{aligned}
$$

Simple but lengthy manipulations lead to the following system

$$
\begin{gathered}
\Lambda_{x x}=0, \quad u_{t} \Lambda_{x u_{t}}-\Lambda_{x}=0, \quad \Lambda_{u_{t} u_{t}}=0, \quad \Lambda_{u}=0, \\
u_{t} \Lambda_{t}+a u_{x} \Lambda_{x}=0, \quad u_{x} \Lambda_{u_{x}}+u_{t} \Lambda_{u_{t}}-\Lambda=0 .
\end{gathered}
$$

The solution of system (18) capitulates the following multiplier

$$
\Lambda\left(t, x, u, u_{t}, u_{x}\right)=\left(-a t d_{3}+d_{2}\right) u_{x}+\left(d_{3} x+d_{1}\right) u_{t},
$$

where $d_{1}, d_{2}$ and $d_{3}$ are arbitrary constants. The choice of constants one by one equal to one and rest to zero yields

$$
\Lambda_{1}=u_{t}, \quad \Lambda_{2}=u_{x}, \Lambda_{3}=-a t u_{x}+x u_{t} .
$$


are

The conserved vectors corresponding to the multipliers (19)

$$
\begin{array}{r}
T_{1}^{t}=\frac{1}{2} u_{t}^{2}-\frac{1}{2} a u_{x}^{2}+\frac{1}{4} c u^{4}+\frac{1}{2} b u^{2}, T_{1}^{x}=a u_{x} u_{t}, \\
T_{2}^{t}=u_{t} u_{x}, T_{2}^{x}=-\frac{1}{2} u_{t}^{2}+\frac{1}{4} c u^{4}+\frac{1}{2} b u^{2}+\frac{1}{2} a u_{x}^{2}, \\
T_{3}^{t}=-\frac{1}{2} a x u_{x}^{2}-a t u_{t} u_{x}+\frac{1}{2} x u_{t}^{2}+\frac{1}{4} c x u^{4}+\frac{1}{2} b x u^{2}, \\
T_{3}^{x}=-\frac{1}{4} a c t u^{4}-\frac{1}{2} a b t u^{2}+\frac{1}{2} a t u_{t}^{2}+a x u_{x} u_{t}-\frac{1}{2} a^{2} t u_{x}^{2} .
\end{array}
$$

Now we apply the symmetry conservation laws relationship to find out which symmetry is associated with conserved vector. Then with the help of double reduction theorem we will determine the exact solution of (15). Eq. (15) possesses the Lie point symmetry generators

$$
X_{1}=\frac{\partial}{\partial t}, \quad X_{2}=\frac{\partial}{\partial x}, \quad X_{3}=-x \frac{\partial}{\partial t}+a t \frac{\partial}{\partial x} .
$$

Exact solution of Eq. (15) using $T_{1}$ : The Lie symmetries $X_{1}$ and $X_{2}$ are associated with the conserved vector $T_{1}$. The reduced conserved form of Eq. (15) can be obtained by using the combination of symmetries $X=X_{1}+\alpha X_{2}$. The canonical form $X=\partial / \partial q$ is derived by solving the characteristic equation

$$
\frac{d t}{1}=\frac{d x}{\alpha}=\frac{d u}{0}=\frac{d r}{0}=\frac{d s}{1}
$$

Thus

$$
s=t, r=x-\alpha t, u=u(r),
$$

where $\alpha$ is a parameter. Making use of (13), we obtain

$$
T_{1}^{r}=\alpha T_{1}^{t}-T_{1}^{x}
$$

Under the change of variables (22) and substituting $T_{1}=$ $\left(T_{1}^{t}, T_{1}^{x}\right)$ from (20), Eq. (23) transforms to

$$
T_{1}^{r}=\frac{\alpha^{3}+a}{2} u_{r}^{2}+\frac{1}{4} \alpha c u^{4}+\frac{1}{2} b \alpha u^{2} .
$$

Since Eq. (24) satisfies $D_{r} T_{1}^{r}=0$ which implies that $T_{1}^{r}=c_{1}$. Replacement of $T_{1}^{r}=c_{1}$ in Eq. (24) to obtain

$$
\frac{\alpha^{3}+a \alpha}{2} u_{r}^{2}+\frac{1}{4} \alpha c u^{4}+\frac{1}{2} b \alpha u^{2}=c_{1} .
$$

The solution of Eq. (25) gives rise to

$$
\pm \int\left(c_{1}-\frac{1}{4} c u^{4}-\frac{1}{2} u^{2}\right)^{-\frac{1}{2}} d u=\sqrt{\frac{2}{\alpha^{3}+a \alpha}}(x-\alpha t)+c_{2},
$$

which constitutes the solution of Eq. (15). Using Maple, we obtain an alternative solution of Eq. (15)

$$
\begin{aligned}
& \frac{1}{\sqrt{c_{1}\left(\alpha b+\sqrt{\alpha\left(\alpha b^{2}+4 c_{1} c\right)}\right)}} \\
& {\left[2 c _ { 1 } J a c o b i S N \left(\frac{\sqrt{\alpha\left(\alpha^{2}+a\right)\left(\alpha b+\sqrt{\alpha\left(\alpha b^{2}+4 c_{1} c\right)}\right)}}{\sqrt{2} \alpha\left(a+\alpha^{2}\right)}\right.\right.} \\
& \left.\frac{\sqrt{-\left(4 c_{1} c+2 b^{2} \alpha+2 b \sqrt{\alpha\left(b^{2} \alpha+4 c_{1} c\right)}\right) c_{1} c}}{2 c_{1} c+b^{2} \alpha+b \sqrt{\alpha\left(b^{2} \alpha+4 c_{1} c\right)}}\right) \\
& r=x-\alpha t .
\end{aligned}
$$

Exact solution of Eq. (15) using $T_{2}$ : Using relation (9) one can easily verify that Lie symmetries $X_{1}$ and $X_{2}$ are associated with the conserved vector $T_{2}$. In terms of canonical coordinates, the reduced form of conserved vector $T_{2}$ is

$$
T_{2}^{r}=\alpha T_{2}^{t}-T_{2}^{x}
$$

Utilizing $T_{2}=\left(T_{2}^{t}, T_{2}^{x}\right)$ from (20) and setting $T_{2}^{r}=c_{1}$ in above equation, we arrive at

$$
\frac{-\alpha^{2}-a}{2} u_{r}^{2}-\frac{1}{2} b u^{2}-\frac{1}{4} c u^{4}=c_{1} .
$$

From Eq. (29), we find the same solution as given in (26).

Remarks: $X_{1}$ is not associated with the conserved vector $T_{3}=\left(T_{3}^{t}, T_{3}^{x}\right)$. Using relation (10), we find that

$T_{3}^{* t}=-a u_{t} u_{x}, \quad T_{3}^{* x}=-\frac{1}{4} a c u^{4}-\frac{1}{2} a b u^{2}+\frac{1}{2} a u_{t}^{2}-\frac{1}{2} a^{2} u_{x}^{2}$

which is a constant multiple of $T_{2}$. Hence in this case we get the same solution of Eq. (15) as derived in Eq. (??). Similarly $X_{2}$ is not associated with $T_{3}=\left(T_{3}^{t}, T_{3}^{x}\right)$ and applying the relation (10), we conclude that

$$
T_{3}^{* * t}=\frac{1}{4} c u^{4}+\frac{1}{2} b u^{2}+\frac{1}{2} u_{t}^{2}-\frac{1}{2} a u_{x}^{2}, T_{3}^{* * x}=a u_{t} u_{x} .
$$

The resulting vector $T_{3}^{* *}$ obtained in Eq. (31) is just a constant multiple of $T_{1}$, so we obtain the same solution of Eq. (15) as in (26).

The vector $X_{3}$ is associated with $T_{3}$, but in terms of canonical

\section{BENJAMIN EQUATION}

Consider the following nonlinear Benjamin equation

$$
u_{t t}+2 a u_{x}^{2}+2 a u u_{x x}+b u_{x x x x}=0,
$$

where $u=u(t, x)$ and $a, b$ are constants. The Benjamin equation describes the dynamics of long internal gravity waves in fluid flow where the surface tension effect cannot be disregarded [10]. The multipliers $\Lambda=\Lambda(t, x, u)$ of Eq. (32) are obtained from the determining equation [18]

$$
E_{u}\left[\Lambda\left(u_{t t}+2 a u_{x}^{2}+2 a u u_{x x}+b u_{x x x x}\right)\right]=0,
$$

where $E_{u}$ is defined in (8). Expansion of Eq. (33) gives rise to

$$
\begin{array}{r}
\Lambda_{u}\left(u_{t t}+2 a u_{x}^{2}+2 a u u_{x x}+b u_{x x x x}\right)+2 a u \Lambda u_{x x} \\
-D_{x}\left[\Lambda\left(4 a u_{x}\right)\right]+D_{t}^{2}[\Lambda]+D_{x}^{2}[\Lambda(2 a u)]+D_{x}^{4}[b \Lambda]=0 .
\end{array}
$$

Equating the coefficients of (34) with respect to different combinations of $u$ yield the following over determined system

$$
\Lambda_{t t}=0, \quad \Lambda_{x x}=0, \quad \Lambda_{u}=0 .
$$

The solution of system (35) established four multipliers

$$
\Lambda_{1}=1, \quad \Lambda_{2}=t, \quad \Lambda_{3}=x, \quad \Lambda_{4}=t x .
$$


Thus we obtain four conservation laws corresponding to multipliers (36)

$T_{1}^{t}=u_{t}, \quad T_{1}^{x}=2 a u u_{x}+b u_{x x x}$,

$T_{2}^{t}=-u+t u_{t}, \quad T_{2}^{x}=2 a t u u_{x}+b t u_{x x x}$,

$T_{3}^{t}=x u_{t}, \quad T_{3}^{x}=2 a x u u_{x}-a u^{2}-b u_{x x}+b x u_{x x x}$,

$T_{4}^{t}=-x u+t x u_{t}, \quad T_{4}^{x}=2 a t x u u_{x}-a t u^{2}-b t u_{x x}+b t x u_{x x x}$.

Eq. (32) possesses the following three symmetries

$$
X_{1}=\frac{\partial}{\partial t}, \quad X_{2}=\frac{\partial}{\partial x}, \quad X_{3}=t \frac{\partial}{\partial t}+\frac{1}{2} x \frac{\partial}{\partial x}-u \frac{\partial}{\partial u} \text {. }
$$

Exact solution of Eq. (32) using $T_{1}$ : We can obtain a reduced conserved form of the PDE by using the association of symmetries with the conserved vectors. From relation (9) it can be easily shown that $X_{1}$ and $X_{2}$ both are associated with $T_{1}$. The generator $X=\frac{\partial}{\partial t}+\alpha \frac{\partial}{\partial x}$ yield $X=\partial / \partial q$ subject to the similarity variables in (22). The $r$-component of $T_{1}$ is then obtained by utilizing (13)

$$
T^{r}=\alpha^{2} u_{r}+2 a u u_{r}+b u_{r r r} .
$$

Setting $T^{r}=c_{1}$ in Eq. (39) gives

$$
\alpha^{2} u_{r}+2 a u u_{r}+b u_{r r r}=c_{1} .
$$

Integration of Eq. (40) with respect to $r$ yields

$$
\alpha^{2} u+a u^{2}+b u_{r r}=c_{1} r+c_{2},
$$

where $c_{1}$ and $c_{2}$ are arbitrary constants. The above equation does not admit any symmetry generator hence it can not be reduced further by using the double reduction theorem [6]. However for particular solution and for sake of simplicity we set $c_{1}=0$ in Eq. (41) to obtain

$$
\alpha^{2} u+a u^{2}+b u_{r r}=c_{2} .
$$

We introduce $u_{r r}$ as $\frac{d u_{r}}{d u} u_{r}$, then the above equation results in

$$
u_{r} d u_{r}=\frac{1}{b}\left(c_{2}-\alpha^{2} u-a u^{2}\right) d u \text {. }
$$

Integration of Eq. (43) leads to

$$
u_{r}= \pm \sqrt{\frac{2}{b}\left(c_{2} u-\frac{1}{2} \alpha^{2} u^{2}-\frac{1}{3} a u^{3}+c_{3}\right)},
$$

which finally yields

$\pm \int \frac{2}{b}\left(c_{2} u-\frac{1}{2} \alpha^{2} u^{2}-\frac{1}{3} a u^{3}+c_{3}\right)^{-\frac{1}{2}} d u=r+c_{4}, \quad r=\alpha t-x$

The above solution satisfies Eq. (32).

Reduction/solution of Eq. (4.1) using $T_{4}$ : The symmetry $X_{3}=t \frac{\partial}{\partial t}+\frac{1}{2} x \frac{\partial}{\partial x}-u \frac{\partial}{\partial u}$ is associated with the conserved vector $T_{4}$ (given in equation (37)). Using Lie symmetry $X_{3}$, we compute the similarity transformation

$$
r=\frac{x}{\sqrt{t}}, \quad s=\ln (t), \quad A(r)=t u
$$

The $r$-component of the conserved vector $T_{4}$ is obtained by utilizing (13) and (46)

$$
T_{4}^{r}=\frac{x}{2} T_{4}^{t}-t T_{4}^{x}
$$

which finally yields

$$
-r^{2} A-\frac{1}{4} r^{3} A_{r}-2 a r A A_{r}+a A^{2}+b A_{r r}-b r A_{r r r}=k_{1},
$$

where we have used $T_{4}^{r}=k_{1}$. Eq. (48) admits the following polynomial solution

$$
A(r)= \pm \frac{\sqrt{a k_{1}+b}}{a}-\frac{r^{2}}{2 a}
$$

which in terms of original variables capitulates

$$
u(t, x)= \pm \frac{\sqrt{a k_{1}+b}}{a t}-\frac{x^{2}}{2 a t^{2}} .
$$

Remarks: $X_{2}$ is associated with $T_{2}$ whereas $X_{1}$ is not. Using relation (10), we obtain $T_{2}^{*}$ which is associated with $X_{1}$ as well as $X_{2}$, but $T_{2}^{*}=T_{1}$, hence we get the same result as in (45).

$X_{1}$ is associated with $T_{3}$ whereas $X_{2}$ is not. From (10), we compute $T_{3}^{*}$ which is associated with $X_{2}$ as well as $X_{1}$, but $T_{3}^{*}=T_{1}$, therefore we obtain the same result derived in (45).

The relationship (9) shows that $X_{1}$ and $X_{2}$ are not associated with $T_{4}$. Employing the same procedure, we obtain $T_{4}^{*}$ which is associated with $X_{1}$ as well as $X_{2}$, but $T_{4}^{*}=T_{1}$, thats why we attain the same result as in (45).

\section{CONSERVATION LAWS AND EXACT SOLUTIONS OF DULLIN-GOTTWALD-HOLM EQUATION}

The integrable shallow water wave equation with linear and non-linear dispersions recognized as the Dullin-GottwaldHolm (DGH) equation $[1,22,23]$

$u_{t}+2 \omega u_{x}+3 u u_{x}-\alpha^{2}\left(u_{t x x}+2 u_{x} u_{x x}+u u_{x x x}\right)+\gamma u_{x x x}=0$

where $\alpha^{2}$ and $\gamma / 2 \omega$ represents square of length scales and $\omega$ denotes the linear speed for undisturbed water at rest and $u(t, x)$ is the fluid velocity. Following two conservation laws of (51) are reported in [1] corresponding to the multiplier $\Lambda=$ $\Lambda\left(t, x, u, u_{t}, u_{x}\right)$ :

$T_{1}^{t}=u-\alpha^{2} u_{x x}, \quad T_{1}^{x}=\frac{3}{2} u^{2}+2 \omega u-\alpha^{2} u u_{x x}-\frac{1}{2} \alpha^{2} u_{x}^{2}+\gamma u_{x x}$

$$
\begin{aligned}
& T_{2}^{t}=\frac{1}{2} u^{2}-\frac{1}{2} \alpha^{2} u_{x}^{2}-\alpha^{2} u u_{x x} \\
& T_{2}^{x}=\omega u^{2}+u^{3}-\alpha^{2} u^{2} u_{x x}+\alpha^{2} u_{t} u_{x}+\gamma\left(u u_{x x}-\frac{1}{2} u_{x}^{2}\right) .
\end{aligned}
$$

Now, we will consider the higher order multipliers $\Lambda=$ $\Lambda\left(t, x, u, u_{t}, u_{x}, u_{x x}\right)$ to compute the associated conservation laws fluxes. The determining equations for multipliers of the form (7) yield

$$
\begin{aligned}
& E_{u}\left[\Lambda \left(u_{t}+2 \omega u_{x}+3 u u_{x}\right.\right. \\
& \left.\left.-\alpha^{2}\left(u_{t x x}+2 u_{x} u_{x x}+u u_{x x x}\right)+\gamma u_{x x x}\right)\right]=0,
\end{aligned}
$$


where $E_{u}$ is the Euler operator presented in (8). Expansion of (53) provides

$$
\begin{aligned}
& \Lambda_{u}\left[\left(u_{t}+2 \omega u_{x}+3 u u_{x}-\alpha^{2}\left(u_{t x x}+2 u_{x} u_{x x}+u u_{x x x}\right)\right.\right. \\
& \left.\left.+\gamma u_{x x x}\right)\right]+\Lambda\left(3 u_{x}-\alpha^{2} u_{x x x}\right)-D_{t}\left[\lambda _ { u _ { t } } \left(u_{t}+2 \omega u_{x}+3 u u_{x}\right.\right. \\
& \left.\left.-\alpha^{2}\left(u_{t x x}+2 u_{x} u_{x x}+u u_{x x x}\right)+\gamma u_{x x x}\right)+\Lambda\right] \\
& -D_{x}\left[\Lambda _ { u _ { x } } \left(u_{t}+2 \omega u_{x}+3 u u_{x}-\alpha^{2}\left(u_{t x x}+2 u_{x} u_{x x}+u u_{x x x}\right)\right.\right. \\
& \left.\left.+\gamma u_{x x x}\right)+\Lambda\left(2 \omega+3 u-2 \alpha^{2} u_{x x}\right)\right] \\
& +D_{x}^{2}\left[\Lambda _ { u _ { x x } } \left(u_{t}+2 \omega u_{x}+3 u u_{x}-\alpha^{2}\left(u_{t x x}\right.\right.\right. \\
& \left.\left.\left.+2 u_{x} u_{x x}+u u_{x x x}\right)+\gamma u_{x x x}\right)+\Lambda\left(-2 \alpha^{2} u_{x}\right)\right] \\
& \quad-D_{x}^{3}\left[\Lambda\left(-\alpha^{2} u+\gamma\right)\right]-D_{x}^{2} D_{t}\left(-\alpha^{2}\right)=0
\end{aligned}
$$

The solution of system (55) for multiplier $\Lambda$ results in

$$
\Lambda=d_{1} u+d_{2}+\frac{d_{3} \alpha^{2} \sqrt{2}}{\sqrt{2 \alpha^{4} u_{x x}-2 \alpha^{2} u-2 \alpha^{2} \omega-\gamma}},
$$

where $d_{1}, d_{2}$ and $d_{3}$ are arbitrary constants. The choice of constants established the following three multipliers

$$
\Lambda_{1}=u, \quad \Lambda_{2}=1, \quad \Lambda_{3}=\frac{\alpha^{2} \sqrt{2}}{\sqrt{2 \alpha^{4} u_{x x}-2 \alpha^{2} u-2 \alpha^{2} \omega-\gamma}} .
$$

The first two multipliers are the same as derived in [1] and the conserved vectors are given in Eq. (52). The new higher order multiplier is related to constant $d_{3}$. Thus the new conserved vector corresponding to the multiplier $\Lambda_{3}$ is

$$
\begin{gathered}
T_{3}^{t}=\sqrt{-\gamma-2 \alpha^{2} \omega}-\sqrt{2 \alpha^{4} u_{x x}-2 \alpha^{2} u-2 \alpha^{2} \omega-\gamma}, \\
T_{3}^{x}=-\sqrt{2 \alpha^{4} u_{x x}-2 \alpha^{2} u-2 \alpha^{2} \omega-\gamma} u-\frac{\gamma}{\alpha^{2}} \sqrt{-\gamma-2 \alpha^{2} \omega} \\
+\frac{\gamma}{\alpha^{2}} \sqrt{2 \alpha^{4} u_{x x}-2 \alpha^{2} u-2 \alpha^{2} \omega-\gamma} .
\end{gathered}
$$

Eq. (51) admits symmetries with generators

$$
\begin{aligned}
& X_{1}=\frac{\partial}{\partial t}, \quad X_{2}=\frac{\partial}{\partial x} \\
& X_{3}=t \frac{\partial}{\partial t}-\left(t w+\frac{3 \gamma}{2 \alpha^{2}} t\right) \frac{\partial}{\partial x}+\frac{(-2 \omega-2 u) \alpha^{2}-\gamma}{2 \alpha^{2}} \frac{\partial}{\partial u}
\end{aligned}
$$

The symmetries $X_{1}$ and $X_{2}$ are associated with $T_{1}, T_{2}$ and $T_{3}$ whereas $X_{3}$ is not associated with any of these conserved vectors.

Exact solution of Eq. (51) using $T_{1}$ : Since $X_{1}$ and $X_{2}$ are associated with $T_{1}$ so the combination of these symmetries $X=X_{1}+\beta X_{2}$ yield the generator $X=\partial / \partial q$ under the change of coordinates

$$
s=x, r=\beta t-x, u=u(r) .
$$

The $r$-component of $T_{1}$ is achieved using (13)

$$
T_{1}^{r}=\beta T_{1}^{t}-T_{1}^{x} .
$$

In terms of canonical variables, Eq. (61) becomes

$$
(\beta-2 w) u-\frac{3}{2} u^{2}+\frac{1}{2} \alpha^{2} u_{r}^{2}+\left(\alpha^{2} u-\beta \alpha^{2}-\gamma\right) u_{r r}=k_{1},
$$

where we have used $T_{1}^{r}=k_{1}$.

The solution of Eq. (62) is

$$
\begin{aligned}
& \pm \int \frac{-\alpha^{2} u+\beta \alpha^{2}+\gamma}{\sqrt{\left(-\alpha^{2} u+\beta \alpha^{2}+\gamma\right)\left(\beta u^{2}-2 u^{2} w-u^{3}-2 k_{1} u+c_{1}\right)}} d u \\
& =r+c_{2}, r=\beta t-x,
\end{aligned}
$$

which comprise the solution of (51). In order to get closed form solution(s) we use sine-cosine method which was presented in [27,28] and is represented below briefly:

\section{Sine-Cosine Algorithm:}

- Integrate the ODE $P\left(u, u_{r}, u_{r r}, \ldots\right)=0$ as many times as possible and set the constants of integration equal zero.

- Consider the solution of the form

$$
u(r)=\lambda \sin (\mu r)^{\kappa},
$$

or

$$
u(r)=\lambda \cos (\mu r)^{\kappa},
$$

where the parameters $\lambda, \mu$ and $\kappa$ are need to be determined.

- $\quad$ Substitute (64) or (65) in $P\left(u, u_{r}, u_{r r}, \ldots\right)=0$ and balance the terms of sine functions when (64) is used or balance the terms of cosine functions when (65) is utilized.

- Separate the terms with respect to powers of sine or cosine functions to obtain algebraic system of equations in terms of $\lambda, \mu$ and $\kappa$ and then solve this system to obtian these parameters.

Employing the above mentioned algorithm to Eq. (62) and setting $k_{1}=0$ we obtain the following two solutions

$$
\begin{array}{ll}
u_{1}(t, x)=-\frac{\gamma+2 w \alpha^{2}}{\alpha^{2}} \cosh ^{2}\left(\frac{\alpha^{2} x+\gamma t}{2 \alpha^{3}}\right), & \beta=-\frac{\gamma}{\alpha^{2}}, \\
u_{2}(t, x)=\frac{\gamma+2 w \alpha^{2}}{\alpha^{2}} \sinh ^{2}\left(\frac{\alpha^{2} x+\gamma t}{2 \alpha^{3}}\right), & \beta=-\frac{\gamma}{\alpha^{2}} .
\end{array}
$$

Exact solution of Eq. (51) using $T_{2}$ : Both the symmetries $X_{1}$ and $X_{2}$ are associated with $T_{2}$. The $r$-component of $T_{2}$ is

$$
T_{2}^{r}=\beta T_{2}^{t}-T_{2}^{x}
$$

If $T_{2}=\left(T_{2}^{t}, T_{2}^{x}\right)$ from (52) is substituted in above equation, then we obtain

$\left(\frac{1}{2} \beta-w\right) u^{2}-u^{3}+\frac{1}{2}\left(\beta \alpha^{2}+\gamma\right) u_{r}^{2}+\left(\alpha^{2} u^{2}-\beta \alpha^{2} u-\gamma u\right) u_{r r}=k_{2}$.

Writing $u_{r r}=\frac{d u_{r}}{d u} u_{r}$ and then integration gives rise to the same solution as obtained in case- 1 above. The closed form solutions of (68) are obtained using sine-cosine algorithm as described above and then the exact solutions of (51) finally expressed as 


$$
\begin{aligned}
& u_{1}(t, x)=-\frac{\gamma+2 w \alpha^{2}}{\alpha^{2}} \cosh ^{2}\left(\frac{x-\beta t}{2 \alpha}\right), \\
& u_{2}(t, x)=\frac{\gamma+2 w \alpha^{2}}{\alpha^{2}} \sinh ^{2}\left(\frac{x-\beta t}{2 \alpha}\right) .
\end{aligned}
$$

Exact solution of Eq. (51) using $T_{3}$ : It can be easily verified that $X_{1}$ and $X_{2}$ are associated with $T_{3}$ hence we obtain the same coordinates as derived in (60). Applying (13), we get the reduced conserved form

$$
\begin{aligned}
& \beta\left(\sqrt{-\gamma-2 \alpha^{2} w}-\sqrt{2 \alpha^{4} u_{r r}-2 \alpha^{2} u-2 \alpha^{2} w-\gamma}\right) \\
& +\sqrt{2 \alpha^{4} u_{r r}-2 \alpha^{2} u-2 \alpha^{2} w-\gamma} u+\frac{\gamma}{\alpha^{2}} \sqrt{-\gamma-2 \alpha^{2} w} \\
& -\frac{\gamma}{\alpha^{2}} \sqrt{2 \alpha^{4} u_{r r}-2 \alpha^{2} u-2 \alpha^{2} w-\gamma}=k_{3}
\end{aligned}
$$

which yields

$$
\begin{aligned}
& \left(-\frac{\gamma}{\alpha^{2}}-\beta+u\right) \sqrt{2 \alpha^{4} u_{r r}-2 \alpha^{2} u-2 \alpha^{2} w-\gamma} \\
& +\left(\frac{\gamma}{\alpha^{2}}+\beta\right) \sqrt{-\gamma-2 \alpha^{2} w}=k_{3}
\end{aligned}
$$

Taking square and then simplification results in

$$
\begin{aligned}
& 2 \alpha^{4} u_{r r}=\frac{\left(k_{3}-\left(\frac{\gamma}{\alpha^{2}}+\beta\right) \sqrt{-\gamma-2 \alpha^{2} w}\right)^{2}}{\left(-\frac{\gamma}{\alpha^{2}}-\beta+u\right)^{2}} \\
& +2 \alpha^{2} u+2 \alpha^{2} w+\gamma .
\end{aligned}
$$

We use $u_{r r}=\frac{d u_{r}}{d u} u_{r}$ in Eq. (73) to obtain

$$
\begin{aligned}
& 2 \alpha^{4} u_{r} \frac{d u_{r}}{d u}=\frac{\left(k_{3}-\left(\frac{\gamma}{\alpha^{2}}+\beta\right) \sqrt{-\gamma-2 \alpha^{2} w}\right)^{2}}{\left(-\frac{\gamma}{\alpha^{2}}-\beta+u\right)^{2}} \\
& +2 \alpha^{2} u+2 \alpha^{2} w+\gamma,
\end{aligned}
$$

and therefore

$$
\begin{aligned}
& \pm \alpha^{2} u_{r}=\left[-\frac{\left(k_{3}-\left(\frac{\gamma}{\alpha^{2}}+\beta\right) \sqrt{-\gamma-2 \alpha^{2} w}\right)^{2}}{\left(-\frac{\gamma}{\alpha^{2}}-\beta+u\right)}\right. \\
& \left.+\alpha^{2} u^{2}+2 \alpha^{2} w u+\gamma u+c_{5}\right]^{\frac{1}{2}} .
\end{aligned}
$$

Integration with respect to $r$ furnishes

$$
\begin{aligned}
& \pm \alpha^{2} \int\left(-\frac{\left(k_{3}-\left(\frac{\gamma}{\alpha^{2}}+\beta\right) \sqrt{-\gamma-2 \alpha^{2} w}\right)^{2}}{\left(-\frac{\gamma}{\alpha^{2}}-\beta+u\right)}\right. \\
& \left.+\alpha^{2} u^{2}+2 \alpha^{2} w u+\gamma u+c_{5}\right)^{-\frac{1}{2}} d u=r+c_{6}, \quad r=\beta t-x,
\end{aligned}
$$

where $c_{5}$ and $c_{6}$ are constants of integration. The above implicit solution satisfies the DGH equation.

\section{Generalized Dullin-GotTwald-Holm EQUATION}

The generalized DGH equation is defined by [24]

$$
\begin{aligned}
& u_{t}+2 w u_{x}+3 u^{m} u_{x}-\alpha^{2}\left(u_{t x x}+2 u_{x} u_{x x}+u u_{x x x}\right) \\
& +\gamma u_{x x x}=0, m>0 .
\end{aligned}
$$

The DGH equation can be derived from the generalized DGH equation by taking $m=1$ and the Camassa-Holm equation can be deduced for $m=1$ and $\gamma=0$. When $\gamma=0$, then Eq. (77) reduces the generalized Camassa-Holm equation considered by Lixin Trian and Xiuying Song [25]. For $\alpha=0$, the generalized $\mathrm{kdv}$ equation is deduced.

The following two conservation laws are derived in [1]:

$$
\begin{aligned}
& T_{1}^{t}=u-\alpha^{2} u_{x x}, \\
& T_{1}^{x}=\frac{3}{m+1} u^{m+1}+2 w u-\alpha^{2} u u_{x x}-\frac{1}{2} \alpha^{2} u_{x}^{2}+\gamma u_{x x}, \\
& T_{2}^{t}=\frac{1}{2} u^{2}-\alpha^{2} u u_{x x}-\frac{1}{2} \alpha^{2} u_{x}^{2}, \\
& T_{2}^{x}=w u^{2}+\frac{3}{m+2} u^{m+2}-\alpha^{2} u^{2} u_{x x}+\alpha^{2} u_{t} u_{x} \\
& +\gamma\left(u u_{x x}-\frac{1}{2} u_{x}^{2}\right) .
\end{aligned}
$$

One can easily verify that for higher order multiplier the variational derivative approach yields the same conservation laws for generalized DGH equation. Eq. (77) admits the following two symmetries

$$
X_{1}=\frac{\partial}{\partial t}, \quad X_{2}=\frac{\partial}{\partial x} .
$$

Employing the same method of solution as in the previous section, the following two solutions are obtained for generalized DGH equation

$$
\begin{aligned}
& \pm \int\left(m^{2} \beta \alpha^{2}+m^{2} \gamma+m \beta \alpha^{2}+3 m \gamma+2 \beta \alpha^{2}+2 \gamma+\right. \\
& \left.\left(-m^{2} \alpha^{2}-3 m \alpha^{2}-2 \alpha^{2}\right) u\right)\left(-\left(m^{2}+3 m+2\right)\right. \\
& \left(6 u^{m+2}-\beta m^{2} u^{2}-3 \beta m u^{2}+2 u^{2} w m^{2}+6 u^{2} w m\right. \\
& +2 u k_{5} m^{2}+6 m k_{5} u-2 u^{2} \beta \\
& \left.+4 u^{2} w+4 k_{5} u-c_{7} m^{2}-3 c_{7} m-2 c_{7}\right) \\
& \left.\left(\beta \alpha^{2}-\alpha^{2} u+\gamma\right)\right)^{-\frac{1}{2}} d u-\beta t+x-c_{8}=0
\end{aligned}
$$

and

$$
\begin{aligned}
& \pm \int\left(m^{2} \beta \alpha^{2}+3 \beta \alpha^{2} m+m^{2} \gamma\right. \\
& +3 m \gamma+2 \beta \alpha^{2}+2 \gamma+\left(-m^{2} \alpha^{2}-3 m \alpha^{2}\right. \\
& \left.\left.-2 \alpha^{2}\right) u\right)\left(-\left(m^{2}+3 m+2\right)\left(4 u^{2} w-u^{2} \beta m^{2}-2 k_{6} m^{2}\right.\right. \\
& -6 k_{6} m-4 k_{6}+6 u^{m+2}-u c_{9} m^{2}-3 u c_{9} m-2 u c_{9}+2 u^{2} w m^{2} \\
& \left.+6 u^{2} w m-3 u^{2} \beta m-2 u^{2} \beta\right) \\
& \left.\left(\beta \alpha^{2}-\alpha^{2} u+\gamma\right)\right)^{-\frac{1}{2}} d u-\beta t+x-c_{10}=0,
\end{aligned}
$$

where $c_{1}, \ldots, c_{10}$ are arbitrary constants.

In case when $m=1$, we obtain the same solution as in (70). The solutions of generalized DGH equation for $m=2$, employing sine-cosine method are

$$
\begin{aligned}
& u_{1}(t, x)=\frac{-\alpha^{2}+\sqrt{(1-4 w) \alpha^{4}-2 \alpha^{2} \gamma}}{\alpha^{2} \cosh ^{2}\left[\sqrt{\frac{\alpha^{2}-\sqrt{(1-4 w) \alpha^{4}-2 \alpha^{2} \gamma}}{8 \alpha^{4}}}(x-\beta t)\right]}, \\
& u_{2}(t, x)=\frac{\alpha^{2}-\sqrt{(1-4 w) \alpha^{4}-2 \alpha^{2} \gamma}}{\alpha^{2} \sinh ^{2}\left[\sqrt{\frac{\alpha^{2}-\sqrt{(1-4 w) \alpha^{4}-2 \alpha^{2} \gamma}}{8 \alpha^{4}}}(x-\beta t)\right]}, \\
& u_{3}(t, x)=\frac{-\alpha^{2}+\sqrt{(1-4 w) \alpha^{4}-2 \alpha^{2} \gamma}}{\alpha^{2} \cos ^{2}\left[\sqrt{\frac{-\alpha^{2}+\sqrt{(1-4 w) \alpha^{4}-2 \alpha^{2} \gamma}}{8 \alpha^{4}}}(x-\beta t)\right]}, \\
& u_{4}(t, x)=\frac{-\alpha^{2}+\sqrt{(1-4 w) \alpha^{4}-2 \alpha^{2} \gamma}}{\alpha^{2} \sin ^{2}\left[\sqrt{\frac{-\alpha^{2}+\sqrt{(1-4 w) \alpha^{4}-2 \alpha^{2} \gamma}}{8 \alpha^{4}}}(x-\beta t)\right]},
\end{aligned}
$$


where

$$
\beta=\frac{\alpha^{2}-\gamma-\operatorname{sqrt}(1-4 w) \alpha^{4}-2 \alpha^{2} \gamma}{\alpha^{2}} .
$$

\section{CONCLUSION}

The conservation laws of non-linear evolution equation, Benjamin equation and DGH equation were derived using multipliers approach. The first order multipliers were considered for non-linear evolution equation and three conserved vectors were constructed. The symmetry conservation law relationship was used to determine symmetries associated with conserved vectors. One exact solution for non-linear evolution equation was computed using the double reduction theorem. For Benjamin equation we considered multiplier as a function of dependent and independent variables and four conserved vectors were obtained. However first order multipliers generated same conserved vectors. The fourth order Benjamin equation was reduced to a second order ODE in terms of similarity variables. A particular solution of this second order ODE was found which constituted the exact solution of Benjamin equation. The double reduction theorem was invoked to compute solutions of DGH equation and generalized DGH equation. The same combination of symmetries $\partial / \partial_{t}$ and $\partial / \partial_{x}$ was used to derive the invariant ansatz for equations under consideration which yielded the same solution by using any associated conserved vector $T_{4}$ and the reduced conserved form of Benjamin equation was derived. The resulting equation could not be reduced further however the polynomial solution was obtained using Maple. Additionally, we invoked the sine-cosine method and the explicit solutions of DGH and generalized DGH equations were established.

\section{REFERENCES}

[1] R. Naz, I. Naeem, S. Abelman, Conservation laws for CammasaHolm equation, Dullin-Gottwald-Holm equation and generalized DullinGottwald-Holm equation, Nonlinear Analysis: Real World Applications, 10, 3466-3471, (2009) .

[2] E. Noether, Invariante Variationsprobleme, Nacr. Konig. Gesell. Wissen., Gottingen, Math.-Phys. Kl. Heft, 2, 235-257, (1918) (English translation in Transport Theory and Statistical Physiscs, 1 (3), 186-207, (1971)).

[3] A. H. Kara, F. M. Mahomed, The Relationship Between Symmetries and Conservation Laws, Int. J. Theor. Phys, 39 (1), 23-40, (2000).

[4] A. Sjöberg, F. M. Mahomed, Non-local Symmetries and Conservation Laws for One-dimensional Gas Dynamics Equations, Appl. Math. Comput., 150 (2), 379-397, (2004).

[5] A. Sjöberg, F. M. Mahomed, The Association of Non-local Symmetries with Conservation Laws: Applications to the Heat and Burgers Equations, Appl. Math. Comput., 168 (2), 1098-1108, (2005).

[6] A. Sjöberg, Double Reduction of PDEs from the Association of Symmetries with Conservation Laws with Applications, Applied Mathematics and Computation, 184, 608-616, (2007).

[7] A. Sjöberg, On Double Reductions from Symmetries and Conservation Laws, Nonlinear Analysis: Real World Applications, 10, 3472-3477, (2009).

[8] A. H. Bokhari, A. Y. Al-Dweik, F. M. Mahomed, F. D. Zaman, Conservation Laws of a Nonlinear $(\mathrm{n}+1)$ Wave Equation, Nonlinear Analysis: Real World Applications, 11, 2862-2870, (2010).

[9] TaoGeTuSang, Sirendaoreji, New type of exact solitary wave solutions for dispersive long-wave equation and Benjamin equation, Acta Phys. Sinica 55, 3246, (2006).

[10] M. J. Ablowitz, P. A. Clarkson, Solitons, Nonlinear Evolution Equations and Inverse Scattering, Cambridge University Press, New York, (1991).

[11] E. G. Fan, H. Q. Zhang, Acta phys. Sinica 46 1244-1254, (1997).
[12] W. Malfliet, Solitary wave solutions of nonlinear wave equations, Amer. J. Phys. 60, 650-654, (1992).

[13] C. H. GU, B. L. GUO, Y. S. LI, Soliton Theory and Its Application, Zhejiang Publishing House of Science and Technology, Hangzhou, 216267, (1990).

[14] R. K. Dodd, J. C. Eilbeck, J. D. Gibbon, H. C. Morries, Solitons and Nonlinear Wave Equations Academic Press, London, (1982).

[15] M. R. Miura, Backlund Transformation, Springer, Berlin, (1978).

[16] A. Jeffrey, M. N. Mohamad, Exact solutions to the KdV-Burgers' equation, Wave Motion, 14 , 369-375, (1990).

[17] H. Steudel, Uber die Zuordnung Zwischen Invarianzeigenschaften und Erhaltungssatzen, Zeit Naturforsch, 17 A, 129-132, (1962).

[18] P. J. Olver, Applications of Lie Groups to Differential Equations, Springer, New York, (1993).

[19] R. Naz, F. M. Mahomed, D. P. Mason, Comparison of Different Approaches to Conservation Laws for Some Partial Differential Equations in Fluid Mechanics, Applied Mathematics and Computation, 205, 212230, (2008).

[20] W. H. Steeb, W. Strampp, Diffusion equations and Lie and Lie-Backlund transformation groups, Physica A 114, 95-99, (1982).

[21] A. H. Kara, F. M. Mahomed, Action of Lie-Backlund symmetries on conservation laws, Modern Group Analysis, VII, Norway, (1997).

[22] H. R. Dullin, G. A. Gottwald, D. D. Holm, An integrable shallow water equation wih linear and nonlinear dispersion, Phys. Rev. Lett. 87 (19), 4501-4504, (2001).

[23] L. Tian, Q. Shi, Boundary control of viscous Dullin-Gottwald-Holm equation, Int. J. Nonlin. Sci. 4(1), 67-75, (2007).

[24] D. Lua, D. Pengb, LTian, On the well-posedness problem for the generalized Dullin-Gottwald-Holm equation, Int. J. Nonlin. SCi. 1 (3), 178-186, (2006).

[25] L. Tian, X. Song, New peaked solitary wave solutions of the generalized Camassa-Holm equation, Chaos Solit. Fract. 19 (3), 621-639, (2004).

[26] R. K. Gupta, Anupma, The Dullin-Gottwald-Holm equation: Classical Lie approach and exact solutions, Int. J. of Nonlin Sci. 10 (2), 146-152, (2010).

[27] A. M. Wazwaz, A sine-cosine method for handling nonlinear wave equations, Math. Comput. Model. 40, 499-508, (2004).

[28] A. M. Wazwaz, The sine-cosine method for obtaining solutions with compact and noncompact structures, Appl. Math. Comput. 159 (2), 559$576,(2004)$.

\section{Creative Commons Attribution License 4.0} (Attribution 4.0 International, CC BY 4.0)

This article is published under the terms of the Creative Commons Attribution License 4.0 https://creativecommons.org/licenses/by/4.0/deed.en_US 